\title{
Sensor-Enabled Geogrids for Stabilization and Performance Monitoring of Earth Structures: State of Development
}

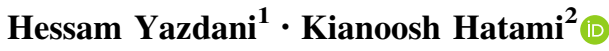

Received: 8 September 2016/Accepted: 2 November 2016/Published online: 9 November 2016

(c) Springer International Publishing Switzerland 2016

\begin{abstract}
The sensor-enabled geogrid (SEGG) technology has been introduced and developed by the authors in the past few years as a new category of geogrid products that possess built-in strain-sensing capability in addition to their conventional reinforcement/stabilization function in geotechnical and transportation applications. The SEGG strain-sensing function arises from their tensoresistivity, which is the sensitivity of their electrical conductivity to tensile strain. This paper reports the state of development of the SEGG technology and reports latest findings on both the in-isolation and in-soil tensoresistivity performance of SEGG specimens. Results indicate that the technology holds promise to serve as an alternative to conventional instrumentation for the performance monitoring of geotechnical structures.
\end{abstract}

Keywords Geosynthetics - Sensors - Smart construction materials $\cdot$ Polymer composites $\cdot$ Structural health monitoring

Kianoosh Hatami

kianoosh@ou.edu

Hessam Yazdani

hessam.yazdani@howard.edu

1 Department of Civil and Environmental Engineering, Howard University, 2300 Sixth Street NW Room 1026, Washington, DC 20059, USA

2 School of Civil Engineering and Environmental Science, The University of Oklahoma, 202 W Boyd St Room 450B, Norman, OK 73019, USA

\section{Introduction}

Structural health monitoring (SHM) and performance assessment are increasingly integrated in modern civil engineering projects to prevent serviceability problems and catastrophic failures. They also help to detect signs of excessive deformation and other problems caused by unpredicted site conditions or other factors during construction, thereby facilitating timely adjustments to complete the construction procedure successfully. Moreover, data collected during the monitoring process can be used to better identify and quantify the uncertainties in the design of earth structures. Consequently, this practice can help improve the reliability and accuracy of related design guidelines, which will in turn lead to more economical designs.

Inherent variability and uncertainties in soil properties of a given project site could pose significant challenges to the design of geotechnical structures including those constructed using geosynthetics. However, more widespread use of SHM and performance evaluation techniques could provide significant help in countering design uncertainties and identifying possible sources of serviceability or stability problems in the structures during their service life.

Strain gauges, optical fibers and extensometers are examples of current technologies that are used to measure strains in geosynthetics where sensing is achieved by attaching these devices to a geosynthetic layer in desirable positions. However, these devices typically require complex and expensive data acquisition systems to collect information. Also, strain gauges attached to a reinforcement material must be calibrated against global strains from crosshead displacements in in-isolation tensile tests. However, the resulting calibration factors are typically not accurate when the reinforcement layer is embedded in soil due to the local stiffening effect of the bonding assembly, 
difference in in-soil mechanical properties relative to the in-isolation values, and other complications such as soil arching.

Significant advancements in sensor and communication technologies during the last decades have brought about significant opportunities for SHM techniques and applications. Examples include highly-accurate 3D point cloud models [1, 2] and extracting structure motions from surveillance videos [3]. In connection with these advancements, in recent years, the authors and their colleagues have developed sensor-enabled geogrids (SEGG) for simultaneous instrumentation and reinforcement/stabilization of earth structures. This paper provides an overview of the latest findings regarding the development of SEGG and discusses the future steps toward its industrialscale production and practical applications.

\section{Geogrids: Types and Applications}

As a major category of geosynthetics, geogrids are polymeric products that are extensively used in civil engineering projects for reinforcement and stabilization applications. Examples include stabilization of highway slopes and embankments [4], and reinforcement of foundations [5, 6], and paved and unpaved roads [7-9] in order to mitigate cracking and rutting, prolong service life and reduce aggregate base course thickness for a given traffic volume.

Geogrids are commonly made of polypropylene (PP), polyethylene (PE) or PVC-coated PET (PVC: polyvinyl chloride). High-tenacity PET yarns are interlaced into an open structure and coated with a CB-filled PVC composite to make woven and knitted geogrids. In comparison, unitized geogrids are made by heat-welding PP or PE strips (bonded geogrids) or stretching needle-punched PP or PE sheets into a grid (extruded geogrids [10]).

\section{Conductive Nanocomposites}

Dispersing a sufficient concentration of conductive particles such as metal nanopowders, carbon blacks (CBs), carbon fibers, carbon nanotubes (CNTs), graphene or synthetic graphite in an insulating polymer matrix results in a conductive composite. Typical changes in the conductivity of a filled composite as a function of the conductive filler concentration are shown in Fig. 1. At low filler concentrations, composite conductivity is close to that of the insulating polymer because the scattered fillers are not close enough to transfer electrons (Stage I). As the filler concentration increases, conductivity of the composite reaches a range of values called the percolation region (Stages II and III) across which a conductive network of

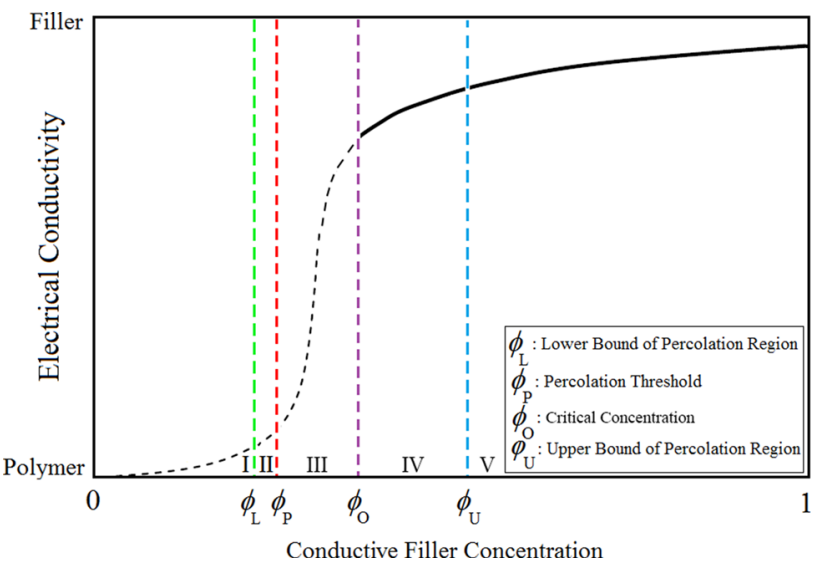

Fig. 1 Filler concentration versus electrical conductivity

fillers is developed. Electrical conductivity of the composite in Stage II is slightly higher than that in Stage I due to the tunneling (hopping) conduction through the thin layers of polymer sandwiched by the fillers [11, 12].

Doping the composite with more fillers causes an excess energy arising from the formation of carbon-polymer interfaces to reach a universal value independent of the polymer type, and then the fillers begin to coagulate to form conductive networks in the composite, from Stage II to Stage III [13]. As the level of doping is increased to a concentration called percolation threshold, a jump in conductivity is observed, implying a transition in the nature of charge transport from tunneling to partial metallic diffusive transport [14]. Adding more filler to the composite would slightly increase its conductivity due to the evolution of the existing or formation of new conductive paths (Stage IV). Beyond the percolation region the conductivity plateaus to a value lower than that of the conductive filler (Stage V). The filler concentration at which the electrical conductivity of a composite would drastically change due to a tensile strain corresponds to the upper bound of the percolation region (transition from Stage III to Stage IV) and is called the critical (target) concentration. This strain-induced change in the electrical conductivity is referred to as tensoresistivity and is represented by the gauge factor, which is defined as the relative change in a specimen's electrical resistance per unit strain, $\varepsilon$, as [15]:

$\mathrm{GF}=\frac{\Delta R / R_{0}}{\varepsilon}$

where $\Delta R$ is change in resistance and $R_{0}$ is unstrained resistance.

\section{SEGG's State of Development}

Sensor-enabled geosynthetic (SEG) technology was first proposed by Hatami et al. [16, 17] who carried out a proofof-concept study to examine the potential of conductive 
polymer composites to add a self-sensing function to conventional geosynthetics. In conventional geosynthetics, carbonaceous fillers (e.g. CB) are typically used for UV protection. However, Hatami et al. [16, 17] added CB and CNT at the corresponding critical concentrations to highdensity PE (HDPE) and PP in order to produce UV-protected and tensoresistive geosynthetic prototypes. The results were promising. With the exception of CNT-filled PP composites, all other composites, especially the CBfilled specimens, exhibited significant tensoresistivity. The mean gauge factors of the composites studied by Hatami et al. $[16,17]$ are listed in Table 1 . The higher sensitivity of CB-filled specimens to tensile strain were attributed to the grape bunch-like configuration of the filler, which compared to the needle-shaped CNTs, would result in a lessentangled network and higher tensoresistivity.

Results of Hatami et al. [16] demonstrated that the SEG technology could lead to a more economical and reliable alternative to commonly used sensors to measure strain (e.g. strain gauges). The SEG technology is cost-effective due to its ability to eliminate the need for complex and expensive data acquisition systems which are normally required for conventional instrumentation. It also could offer improved accuracy in registering the strain. The calibration factors of strain gauges are typically obtained using in-isolation tensile tests. However, the soil confining pressure and interlocking effects could introduce significant errors in these factors and, consequently, in the measured geosynthetic strains in the field. In addition, the strain gauges bonded to a geosynthetic layer typically create a local "hard spot" that causes the under-registration of global tensile strains, potentially making the detection of and accommodation for impending failures futile [18, 19]. The SEG technology avoids these drawbacks by integrating the self-sensing function in the products.

The pioneering studies of Hatami et al. [16, 17] set the stage for a long-term research plan on SEG development. Fathi et al. [20] investigated the influence of CB type on the percolation threshold, mechanical properties and tensoresistivity of low-density PE (LDPE) and PP composites for potential use in unitized SEGG. CB is an amorphous form of carbon and is formed when aromatic hydrocarbons are subjected to incomplete combustion at high temperatures. The structure of $\mathrm{CB}$ is determined per the particle size, aggregate size and shape of its primary particles. Smaller primary particles have higher inter-aggregate attractive forces, resulting in a $\mathrm{CB}$ with higher structure and larger agglomerate size. A low-structure $\mathrm{CB}$, in contrast, is formed when the aggregates are composed of few primary particles [21]. In addition to its UV-resistivity, another advantage of using CB in SEGG is its very low cost relative to the total cost of geogrid. Therefore, a small adjustment in $\mathrm{CB}$ concentration in the existing formulations of geogrids (e.g. 1-4\%) is not expected to have a noticeable influence on their production cost, nor does it require a considerable modification to the existing production processes currently employed by the geogrid manufacturers.

Findings of Fathi et al. [20] supported the hypothesis that the critical concentration of carbon blacks is inversely proportional to their structure. With respect to particle size, their findings indicated that the composites containing larger CB particles would exhibit higher tensoresistivity. Compared to LDPE composites, a higher tensoresistivity was observed in the PP composites (Table 2). Irrespective of the host polymer used, unitized SEGG specimens generally exhibited significantly higher gauge factors compared to typical commercial strain gauges (e.g. $\mathrm{GF}=2$ ).

However, germane to mechanical properties, a $\mathrm{CB}$ concentration (by weight) as high as $20 \%$ reduced the tensile strength of the PP composites by $50 \%$ while leaving the LDPE composites' tensile strength practically unchanged. This more pronounced reduction in the tensile strength of PP could be due to its higher crystallinity (70-80\% for PP vs. $45-55 \%$ for LDPE-[22]). It has been shown that adding $30 \% \mathrm{CB}$ to PP decreases its crystallinity to nearly $33 \%$ [23]. This is due to $\mathrm{CB}$ particles and agglomerates breaking the weak chemical bonds between monomers, which in turn promotes the polymer amorphous regions and reduces its strength [24]. Fathi's results also indicated a sizeable reduction in the strain at failure of both LDPE and PP composites upon adding a small amount of CB.

Recent research toward SEG development has mostly been focused on woven and knitted SEGG. As stated earlier, CB-filled PVC is a polymer composite that is commonly used as the coating of woven and knitted geogrids. $\mathrm{PVC}$ is a thermoplastic insulating polymer with a relatively
Table 1 Mean gauge factors obtained for CB- and CNTfilled HDPE and PP [16]

\begin{tabular}{lllll}
\hline Polymer & Filler & $\begin{array}{l}\text { Critical concentration } \\
(\mathrm{wt} \%)\end{array}$ & $\begin{array}{l}\text { Maximum strain } \\
\text { applied }(\%)\end{array}$ & Mean GF \\
\hline HDPE & CB & 50 & 15 & 20 \\
& CNT & 4.38 & 15 & 7 \\
PP & CB & 33 & 5 & 25 \\
& CNT & 2.8 & 4 & 0.5 \\
\hline
\end{tabular}


Table 2 Mean gauge factors obtained for LDPE and PP composites filled with varyingstructure CBs [20]

\begin{tabular}{lllll}
\hline Polymer & CB structure & $\begin{array}{l}\text { Critical concentration } \\
(\text { wt } \%)\end{array}$ & $\begin{array}{l}\text { Maximum strain } \\
\text { applied (\%) }\end{array}$ & Mean GF \\
\hline LDPE & Low & 10 & 10 & $3-15$ \\
& Moderate & 5 & 10 & 10 \\
& High & 5 & 10 & 9 \\
PP & Low & 7 & 4 & 25 \\
& Moderate & 7 & 10 & 17 \\
\hline
\end{tabular}

low degree of crystallinity. Similar to PP, this means that adding CB to PVC even at low concentrations could result in the deterioration of the composite's mechanical properties including its strength, elastic modulus and ductility. Therefore, the CB concentration in PVC composites should be kept at a minimum. At the same time, a relatively high $\mathrm{CB}$ concentration (e.g. $5 \mathrm{wt} \%$ ) is typically required to make PVC conductive. These conflicting requirements should be addressed simultaneously through a controlled fabrication process that would involve proper mixing, molding and curing stages. An advantage of PVC-coated PET yarn SEGG is that in these products the inner woven PET yarns are the actual load-bearing component, and therefore any changes in the tensile strength of the coating due to increased CB concentration will not adversely influence the tensile properties of the SEGG reinforcement product. Nevertheless, the coating composite is required to retain its integrity in order to maintain its function of protecting PET against the detrimental effects of installation damage and the surrounding environment (i.e. bioactive and other aggressive materials such as aqueous solutions of salts, acids and alkalis as well as any electrical interference from wet soils).

Hatami et al. [25] were the first to investigate the tensoresistivity and mechanical properties of CB-filled PVC composites for their potential use in the production of SEGG. They blended controlled concentrations of low- and moderate-structure $\mathrm{CBs}$ in powder form with plasticized PVC to produce PVC/PET SEGG composites. Both types of CB-filled composites exhibited adequate in-isolation strain sensitivity for potential use in field applications. However, similar to PP and LDPE [20] and as shown in Fig. 2a and b, low-structure black PVC composites exhibited higher tensoresistivity because the conductive network was more prone to rupture and discontinuities under tensile load.

The studies reviewed above primarily investigated the strain-conductivity performance of SEG materials under monotonic loading. However, geogrids are commonly used to stabilize a wide range of earthwork structures that are subjected to time-dependent loading (e.g. traffic). This type of loading could result in irreversible deformations in the CB conductive network of SEGG and alter their electrical

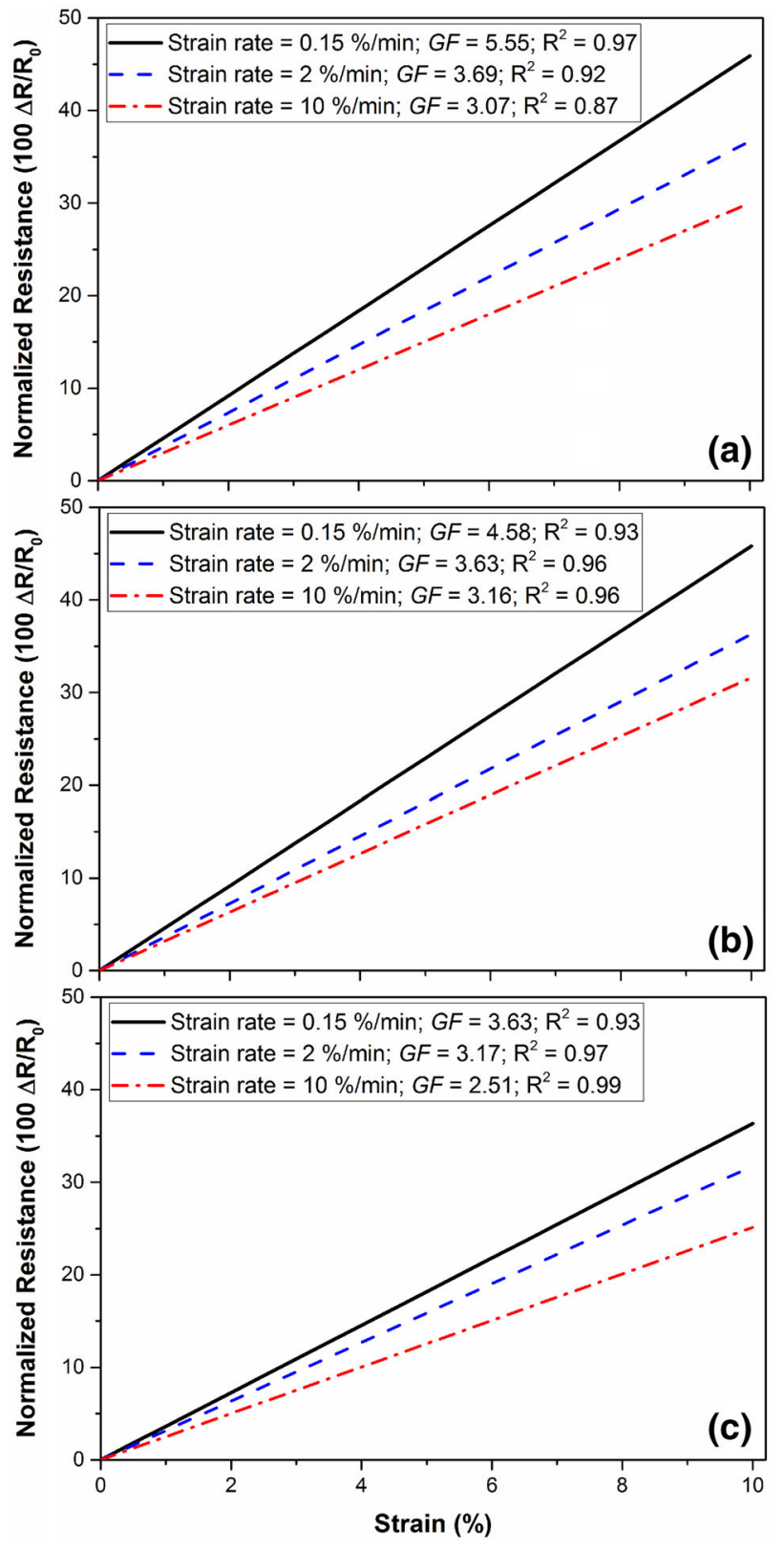

Fig. 2 Tensoresistivity response of PVC composites filled with a low-structure $\mathrm{CB}, \mathbf{b}$ moderate-structure $\mathrm{CB}$ and $\mathbf{c} \mathrm{CNT}$ ( $G F$ gauge factor, Eq. 1) [25, 34]

conductivity. Yazdani et al. [26] used the same CBs as those used by Hatami et al. [25] to study the influence of cyclic loading on the tensoresistivity of $\mathrm{CB} / \mathrm{PVC}$ 
composites. They investigated the influences of factors such as strain magnitude, stress relaxation, loading rate and prestraining on the cyclic performance of $\mathrm{CB} / \mathrm{PVC}$ composites. Yazdani et al.'s results indicated that the conductive network in coating specimens filled with a higherstructure CB experienced less damage upon cyclic deformation and showed more resilience in their tensoresistivity response. Prestraining of coating specimens also improved the resilience in strain sensitivity. The degree of prestraining that was applied to the specimens was quantified by the ratio of initial peak strain to the subsequent operating peak strain. A greater ratio resulted in a more stable and consistent resilience in the tensoresistivity response of the composite.

The studies on SEGG reviewed above were solely focused on the in-isolation performance of both unitized and coated yarn SEGG prototypes. However, the tensoresistivity performance of SEGG is expected to be influenced by the soil confining pressure in field applications. Yazdani et al. [27] modified a standard direct shear test apparatus and used it to investigate the influence of confining pressure on the tensoresistive response of PVC-coated PET yarn SEGG. They carried out their in-soil tensile tests at three different confining pressures of 10,30 and $50 \mathrm{kPa}$ to represent upper levels of reinforced soil structures where larger deformations typically occur [4]. It was found that greater confining pressures and strain rates both reduce the tensoresistivity of SEGG samples (Fig. 3). However, both the magnitude and reproducibility of the measured tensoresistivity in the in-soil tests were considered to be acceptable for civil engineering applications.

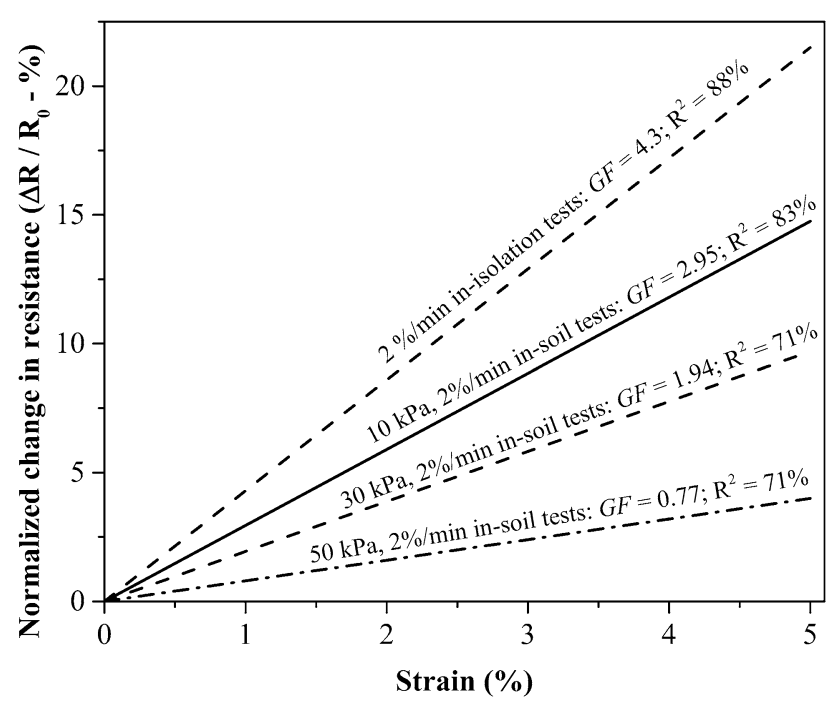

Fig. 3 In-isolation and confined normalized resistance of SEGG specimens as a function of strain (strain rate $=2 \% / \mathrm{min}$; confining pressures $=10,30$ and $50 \mathrm{kPa}$ )
Research on the SEGG development has recently found new prospects, thanks to a continuous and significant drop in the price of carbon nanotubes from approximately $\$ 1,500,000$ per kilogram in 1999 to retail prices in the range of $\$ 50-\$ 300$ per kilogram today [28]. CNTs are increasingly used in nanocomposites due to their highly desirable characteristics such as high flexibility, low density and large aspect ratio (length-to-diameter ratio) in addition to exceptional mechanical and electrical properties. The use of CNTs in SEGG is promising yet challenging due to some difficulties in their proper dispersion in polymers. The term dispersion generally refers to the nearly-uniform distribution of individual fillers in a matrix. However, this definition should be adjusted for such fillers as CNTs that possess an inherent thermodynamic drive to create physical entanglement with neighboring tubules, thus forming aggregated morphologies called bundles. Each bundle is comprised of hundreds of tightly-packed CNTs, which are bound to each other by van der Waals attraction energies of approximately $500 \mathrm{eV} / \mu \mathrm{m}$ per CNTCNT contact [29]. This entanglement tendency is particularly strong due to the generally high aspect ratio and flexibility of CNTs [30].

Improving the quality of CNT dispersion in a polymer would require a trade-off between the electrical and mechanical properties of the resulting composite. On the one hand, a perfect dispersion results in smaller bundles and therefore smaller stress concentration zones that could otherwise compromise the weight, mechanical properties and manufacturability of a composite [31]. On the other hand, bundling has been shown to reduce the percolation threshold [32]. Because the mechanical and electrical properties of SEGG are equally important (especially for unitized SEGG), it is important to devise an optimum mixing and fabrication procedure that would result in a desired level of CNT bundling. It follows that identifying efficient dispersion techniques and characterizing the resulting dispersion quality are essential (albeit challenging) steps in the design of CNT-polymer composites.

Yazdani et al. [33, 34] investigated the influences of dispersion quality and processing conditions on the electrical and mechanical properties of multi-walled CNT-filled PVC (MWCNT/PVC) composites for potential use in SEGG and other applications involving electrically-conductive polymer composites. They measured the electrical conductivity and mechanical properties of the samples that were made using different mixing techniques and quantified subsurface dispersion in the samples using a novel technique which was developed by Smith et al. [35]. Their findings indicated that samples with better quality of dispersion exhibited greater ultimate strength and failure strain, whereas poorly-dispersed specimens showed greater elastic modulus values. 
Yazdani [36] studied the tensoresistivity and mechanical properties of MWCNT/PVC composites subjected to tensile loading and showed that adding $0.5 \mathrm{wt} \%$ MWCNT to the composite results in a considerable reduction in its failure strain and a five-fold increase in its tensile modulus while leaving its ultimate strength almost unchanged. Gauge factors greater than those of typical foil strain gauges and conventional metals were obtained for MWCNT/PVC SEGG (Fig. 2c), indicating their strong potential for different structural performance monitoring and damage detection applications.

It is worth noting that, by and large, in civil engineering applications, geogrids are installed in sufficient depth such that their temperature does not change significantly during service. Therefore, it is considered safe to assume that the strain sensitivity of SEGG products will not be adversely affected by temperature in typical applications.

In continuation of the development of the SEGG technology, a next step would be to design a durable, nonconductive yet UV-protective shield for SEGG products in order to electrically insulate them from the interference of surrounding environment, and to minimize installation damage.

\section{Conclusions}

The state of development of the SEGG technology was presented, and latest findings on the in-isolation and in-soil strain sensitivity performances of SEGG specimens under monotonic and cyclic loading were reviewed. Research on the SEGG technology has so far yielded promising results showing its potential as a practical and cost-effective alternative to existing technologies for the performancemonitoring of geotechnical structures. For instance, compared to strain gauges that require expensive data acquisition systems that prevent their widespread use in field projects, the SEGG technology is amenable to measuring and monitoring strains in large numbers in geosynthetic layers (e.g. geogrid reinforcement), providing significantly larger numbers of data points including e.g. those at comparable locations within the structure that can help to significantly improve the accuracy and reliability of the performance data. Also, for applications involving cyclic loading (e.g. traffic load), SEGG specimens containing higher-structure carbon blacks have been shown to experience less damage and exhibit more resilience in their strain sensitivity response.

Preliminary studies on in-soil tensoresistivity performance of SEGG specimens have been promising. Ongoing research on this technology includes more detailed study of their in-soil performance and addressing practical issues such as durability and protective measures for field installation before they can be pilot-tested in field projects.

\section{References}

1. Khaloo A, Lattanzi D (2016) Hierarchical dense structure-frommotion reconstructions for infrastructure condition assessment. J Comput Civ Eng: 4016047. doi:10.1061/(ASCE)CP.1943-5487. 0000616

2. Khaloo A, Lattanzi D (2015) A Hierarchical computer vision approach to infrastructure inspection. In: Comput. Civ. Eng. 2015. American Society of Civil Engineers, pp 540-547

3. Khaloo A, Lattanzi D (2016) Repurposing video recordings for structure motion estimations. In: SPIE 9805 Health Monit. Struct. Biol. Syst. Las Vegas, NV, pp 980410-980410-7

4. Hatami K, Bathurst RJ (2005) Development and verification of a numerical model for the analysis of geosynthetic-reinforced soil segmental walls under working stress conditions. Can Geotech J 42:1066-1085. doi:10.1139/t05-040

5. Hatami K, Raymond G (2006) Influence of soil strength on optimal embedment depth of geosynthetic reinforcement layer in granular-reinforced foundations. Transp Res Rec 1975:155-162. doi: $10.3141 / 1975-19$

6. Biswas A, Ansari MA, Dash SK, Krishna AM (2015) Behavior of geogrid reinforced foundation systems supported on clay subgrades of different strengths. Int J Geosynth Ground Eng 1:20. doi:10.1007/s40891-015-0023-5

7. Giroud JP, Han J (2004) Design method for geogrid-reinforced unpaved roads. I. Development of design method. J Geotech Geoenviron Eng 130:775-786. doi:10.1061/(ASCE)10900241(2004)130:8(775)

8. Giroud JP, Han J (2004) Design method for geogrid-reinforced unpaved roads. II. Calibration and applications. J Geotech Geoenviron Eng 130:787-797. doi:10.1061/(ASCE)10900241(2004)130:8(787)

9. Ling H, Liu Z (2001) Performance of geosynthetic-reinforced asphalt pavements. J Geotech Geoenviron Eng 127:177-184. doi:10.1061/(ASCE)1090-0241(2001)127:2(177)

10. Koerner RM (2012) Designing with geosynthetics, vol 1, 6th edn. Xlibris Corp, Bloomington

11. Sarychev AK, Brouers F (1994) New scaling for ac properties of percolating composite materials. Phys Rev Lett 73:2895-2898. doi:10.1103/PhysRevLett.73.2895

12. Alamusi HuN, Fukunaga $\mathrm{H}$ et al (2011) Piezoresistive strain sensors made from carbon nanotubes based polymer nanocomposites. Sensors 11:10691-10723. doi:10.3390/s111110691

13. Miyasaka K, Watanabe K, Jojima E et al (1982) Electrical conductivity of carbon-polymer composites as a function of carbon content. J Mater Sci 17:1610-1616. doi:10.1007/BF00540785

14. Du F, Scogna RC, Zhou W et al (2004) Nanotube networks in polymer nanocomposites: rheology and electrical conductivity. Macromolecules 37:9048-9055. doi:10.1021/ma049164g

15. Aneli JN, Zaikov GE, Khananashvili LM (1999) Effects of mechanical deformations on the structurization and electric conductivity of electric conducting polymer composites. J Appl Polym Sci 74:601-621. doi:10.1002/(SICI)10974628(19991017)74:3<601:AID-APP14>3.0.CO;2-K

16. Hatami K, Grady BP, Ulmer MC (2009) Sensor-enabled geosynthetics: use of conducting carbon networks as geosynthetic sensors. J Geotech Geoenviron Eng 135:863-874. doi:10.1061/ (ASCE)GT.1943-5606.0000062

17. Hatami K, Grady B, Ulmer M (2011) Closure to "sensor-enabled geosynthetics: use of conducting carbon networks as geosynthetic 
sensors" by Kianoosh Hatami, Brian P. Grady, and Matthew C. Ulmer. J Geotech Geoenviron Eng 137:435-436. doi:10.1061/ (ASCE)GT.1943-5606.0000472

18. Bathurst RJ, Allen TM, Walters DL (2002) Short-term strain and deformation behavior of geosynthetic walls at working stress conditions. Geosynth Int 9:451-482. doi:10.1680/gein.9.0225

19. Bathurst RJ, Allen TM, Walters DL (2005) Reinforcement loads in geosynthetic walls and the case for a new working stress design method. Geotext Geomembr 23:287-322. doi:10.1016/j.geo texmem.2005.01.002

20. Fathi A, Hatami K, Grady BP (2012) Effect of carbon black structure on low-strain conductivity of polypropylene and lowdensity polyethylene composites. Polym Eng Sci 52:549-556. doi:10.1002/pen.22115

21. Medalia AI (1986) Electrical Conduction in carbon black composites. Rubber Chem Technol 59:432-454. doi:10.5254/1. 3538209

22. Ehrenstein GW (2001) Polymeric materials: structure, properties, applications. Hanser Publishers, Cincinnati

23. Kanbur Y, Küçükyavuz Z (2009) Electrical and mechanical properties of polypropylene/carbon black composites. J Reinf Plast Compos 28:2251-2260. doi:10.1177/0731684408092378

24. Al-Saleh MH, Sundararaj U (2008) Electromagnetic interference (EMI) shielding effectiveness of PP/PS polymer blends containing high structure carbon black. Macromol Mater Eng 293:789. doi:10.1002/mame.200800222

25. Hatami K, Hassanikhah A, Yazdani H, Grady B (2014) Tensoresistive PVC coating for sensor-enabled geogrids. J Nanomech Micromech 4:A4013016. doi:10.1061/(ASCE)NM.2153-5477. 0000070

26. Yazdani H, Hatami K, Khosravi E et al (2014) Strain-sensitive conductivity of carbon black-filled PVC composites subjected to cyclic loading. Carbon 79:393-405. doi:10.1016/j.carbon.2014. 07.082
27. Yazdani H, Hatami K, Grady BP (2016) Sensor-enabled geogrids for performance monitoring of reinforced soil structures. J Test Eval 44:20140501. doi:10.1520/JTE20140501

28. Cheap tubes (2015). In: Cheap tubes. https://www.cheaptubes. com/. Accessed 7 Sep 2016

29. Thess A, Lee R, Nikolaev P et al (1996) Crystalline ropes of metallic carbon nanotubes. Science 273:483-487. doi:10.1126/ science. 273.5274 .483

30. Lourie O, Cox DM, Wagner HD (1998) Buckling and collapse of embedded carbon nanotubes. Phys Rev Lett 81:1638-1641. doi:10.1103/PhysRevLett.81.1638

31. Ajayan PM, Schadler LS, Giannaris C, Rubio A (2000) Singlewalled carbon nanotube-polymer composites: strength and weakness. Adv Mater 12:750-753. doi:10.1002/(SICI)15214095(200005)12:10<750:AID-ADMA750>3.0.CO;2-6

32. Grady BP (2010) Recent developments concerning the dispersion of carbon nanotubes in polymers. Macromol Rapid Commun 31:247-257. doi:10.1002/marc.200900514

33. Yazdani H, Smith BE, Hatami K (2016) Multi-walled carbon nanotube-filled polyvinyl chloride composites: influence of processing method on dispersion quality, electrical conductivity and mechanical properties. Compos Part Appl Sci Manuf 82:65-77. doi:10.1016/j.compositesa.2015.12.005

34. Yazdani H, Smith BE, Hatami K (2016) Electrical conductivity and mechanical performance of multiwalled CNT-filled polyvinyl chloride composites subjected to tensile load. J Appl Polym Sci. doi:10.1002/app.43665

35. Smith B, Yazdani H, Hatami K (2015) Three-dimensional imaging and quantitative analysis of dispersion and mechanical failure in filled nanocomposites. Compos Part Appl Sci Manuf 79:23-29. doi:10.1016/j.compositesa.2015.08.019

36. Yazdani H (2015) Laboratory development and molecular-scale simulation of sensor-enabled geogrids. The University of Oklahoma 